\title{
Entrelacs
}

Cinéma et audiovisuel

\section{Matthew Barney, artiste/auteur : pour une hybridation des genres}

\section{Marie-Laure Delaporte}

\section{(2) OpenEdition}

\section{Journals}

Édition électronique

URL : http://journals.openedition.org/entrelacs/343

DOI : 10.4000/entrelacs.343

ISSN : 2261-5482

Éditeur

Éditions Téraèdre

Référence électronique

Marie-Laure Delaporte, « Matthew Barney, artiste/auteur : pour une hybridation des genres », Entrelacs [En ligne], 9 | 2012, mis en ligne le 22 octobre 2012, consulté le 01 mai 2019. URL : http:// journals.openedition.org/entrelacs/343; DOI : 10.4000/entrelacs.343

Ce document a été généré automatiquement le 1 mai 2019.

Tous droits réservés 


\title{
Matthew Barney, artiste/auteur : pour une hybridation des genres ${ }^{1}$
}

\author{
Marie-Laure Delaporte
}

Dès ses premières œuvres, l'artiste américain Matthew Barney exhibe son corps d'athlète aux prises avec des machines dans des épreuves physiques d'endurance et de douleur témoignant d'un contrôle et d'une puissance sur le corps de l'artiste. Au-delà de son identité, c'est «la recherche des principes fondamentaux de l'identité humaine $»^{2}$. Dans chacune de ses œuvres Barney se travestit et adopte l'identité d'un personnage afin d'exécuter sa performance, et plus particulièrement dans ses deux séries Drawing Restraint (1987-2009) et The Cremaster Cycle (1992-2002).

2 La pratique de Matthew Barney connaît certains précédents dans les générations d'artistes de la seconde moitié $\mathrm{du} \mathrm{XX}^{\mathrm{e}}$ siècle : l'utilisation du corps comme véhicule artistique et l'introduction de la performance et de matériaux viscéraux comme médiums expressifs chez Joseph Beuys, Vito Acconci ou Carolee Schneemann. De même, les actions de Chris Burden ou Marina Abramovic, qui dépeignent l'artiste en quasi-héros et utilisent la vidéo pour sa nature autosuggestive ainsi que le corps comme moyen de persévérance et de transcendance, sont à mettre en relation directe avec la pratique de Matthew Barney ${ }^{3}$. Mais d'autres influences plus populaires sont sous-jacentes dans son œuvre, telles que la transformation et la fusion de l'homme inspirées des films d'horreur, ainsi que les retransmissions télévisuelles de rencontres sportives ou encore les clips musicaux. Pour autant d'images que l'artiste propose, il est possible de trouver autant d'allusions à l'histoire de l'art, de Bronzino à Böcklin, au néo-gothique anglais, au symbolisme de fin de siècle, à Chirico ou à L'Âge d'or de Bunuel et Dali ${ }^{4}$. Cette présence du surréalisme peut aussi être décelée dans la juxtaposition d'éléments hétéroclites créant un effet d'étrangeté, dans les passages d'un état de rêve à celui de réel et une utilisation quasiment fétichiste des costumes et des objets. L'extravagance de Barney vis-à-vis des genres fixés et son exploration du glissement entre masculin et féminin ne sont pas sans rappeler le jeu érotique initié par Marcel Duchamp $p^{5}$. La pratique de la forme artistique qu'est la performance témoigne de la volonté de l'artiste d'un contrôle total sur son corps et de sa perception dans un système cyclique qui lui permettrait de réaffirmer son 
identité à la fois artistique mais aussi sa masculinité après les discours féministes des années 1960 et 1970. Dans la droite ligne des performers tels que Bruce Nauman, une part de l'expression artistique de Barney est susceptible de s'inscrire dans les recherches des gender studies comme celles de Judith Butler ou Griselda Pollock ${ }^{6}$.

\section{De la vidéo des corps}

3 La thématique que Matthew Barney se propose de renouveler est celle déjà abordée dans les décennies 1960 et 1970 par les artistes utilisant leur corps comme véhicule de sens et la vidéo pour en enregistrer les événements. Cette pratique renouvelle la relation entre l'action, l'objet et la vidéo. La notion de contrainte est le point focal autour duquel Matthew Barney développe la superposition du rôle créateur de l'artiste et de l'athlète. (Fig .1) Dans la série Drawing Restraint, débutée en 1987, il s'impose des obstacles afin de compliquer, voire de rendre impossible l'acte de dessiner. Mais ces expérimentations de studio, si elles sont à l'origine des futures performances, ne mettent en scène aucun personnage, uniquement l'artiste testant des processus de création permettant de développer une forme artistique, mais se focalisant davantage sur le processus que sur la création finie qui relève souvent de la forme du schéma ou de l'esquisse.

Fig. 1 : Matthew Barney, DRAWING RESTRAINT 2, 1988

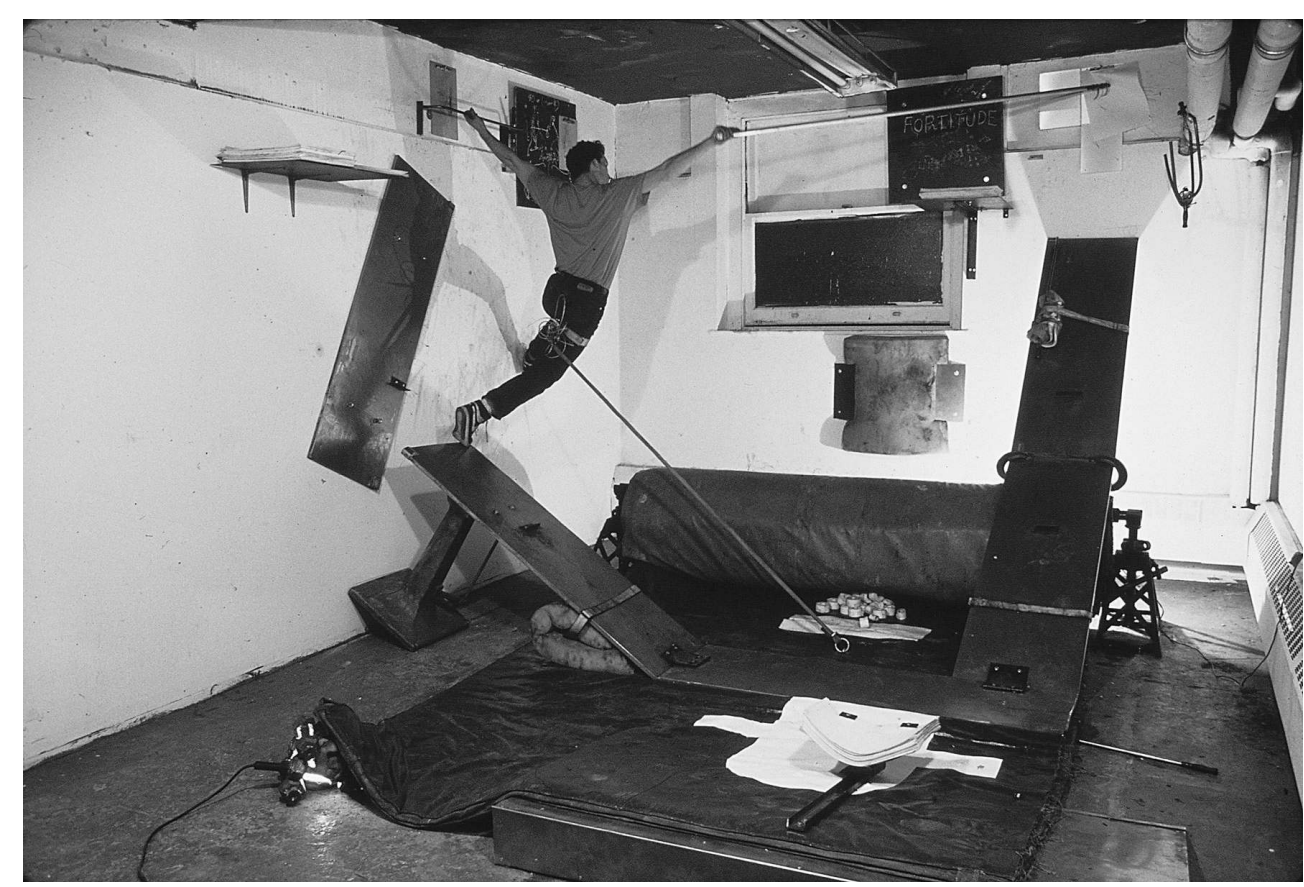

ACTION ET VUE D'INSTALLATION

Copyright Matthew Barney 1988

Photo: Michael Rees

Courtesy Gladstone Gallery, New York

4 Dans les six premiers épisodes, entre 1987 et 1989, il s'engage dans des actions en studio dans lesquelles il construit des obstacles avec des rampes, des trampolines, des élastiques pour s'auto-contraindre dans l'acte créatif. Élaborées en privé, ces expérimentations sont filmées et/ou photographiées afin de conserver une trace documentaire en noir et blanc de ces événements éphémères. Pourtant cet usage adopte une forme hybride entre 
documentaire et installation. Relevant d'une esthétique de style documentaire, certains travaux relèvent presque du domaine domestique et laissent croire à une action réelle et non artificielle.

\section{Vers un nouveau lieu spatio-temporel de création}

5 (Fig.2) Exposée en 1989, à la galerie Althea Viafora de New York, Field Dressing (orifill) est l'une des premières performances dans laquelle Matthew Barney se met en scène, et plus précisément dans un personnage d'athlète, appelé le "Character of Positive Restraint " (personnage de la contrainte positive) inspiré du prestidigitateur Harry Houdini et annonçant le concept de The Path (le chemin). Il trouve son incarnation dans les performances et les vidéos de l'année 1991: Blind Perineum, Transexualis et MILE HIGH Threshold: Flight with the anal sadistic warrior (Seuil à un mile de hauteur: vol avec le guerrier anal-sadique), présentées dans les galeries Barbara Gladstone de New York et Regen Projects de Los Angeles. Ces dernières expositions compliquent quelque peu le champ temporel car elles présentent plusieurs vidéos et sculptures aux titres différents au sein d'une même installation.

Fig. 2 : Matthew Barney, FIELD DRESSING, 1989

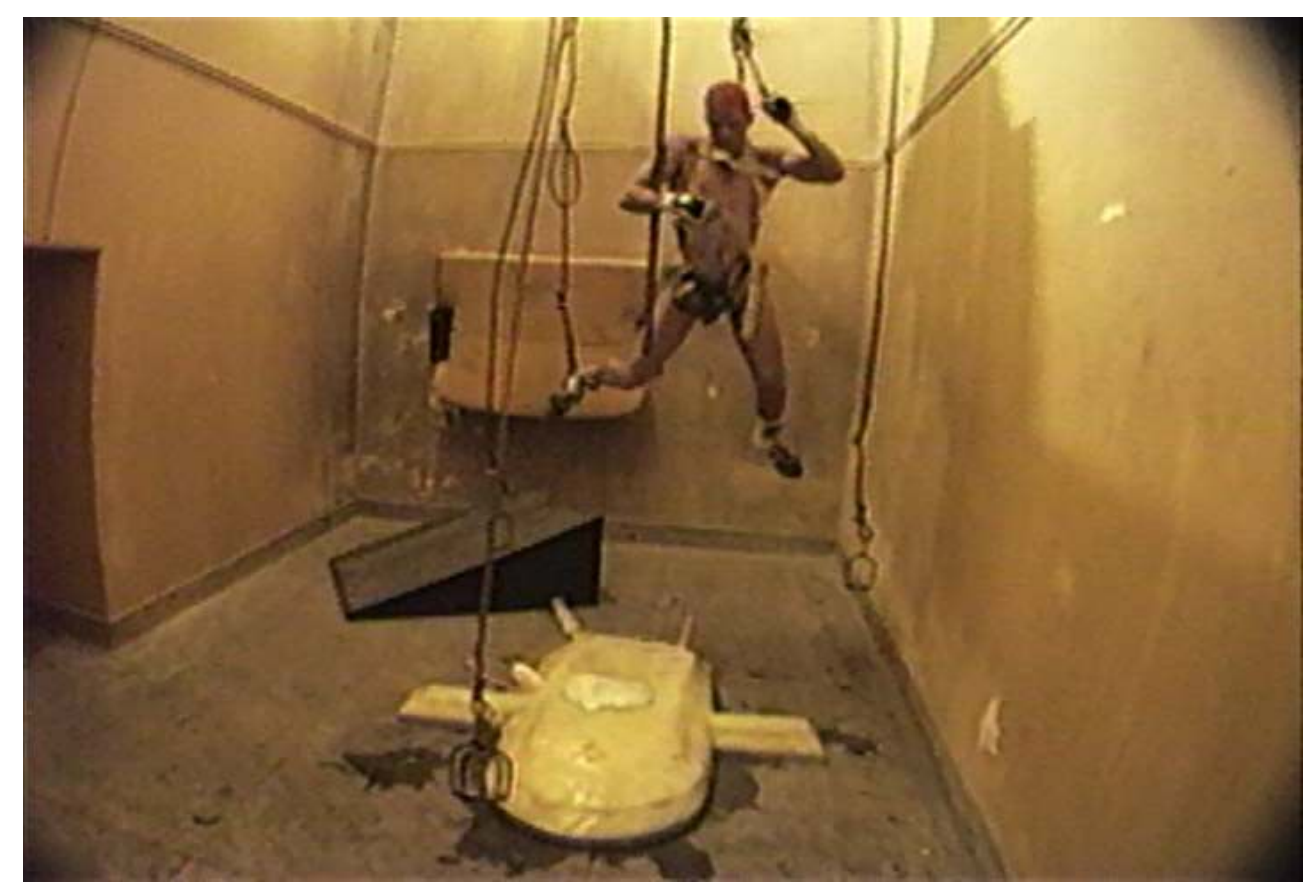

VUE D'INSTALLATION

Payne Whitney Gymnasium, Yale University

Copyright Matthew Barney

Courtesy Gladstone Gallery, New York

Ces actions se déroulent toutes en privé, dans un silence portant l'atmosphère de concentration de l'artiste, toujours nu, hormis ses accessoires (harnais, pics à glace...) l'aidant à escalader. Les pièces dans lesquelles se déroulent les actions abritent également des sculptures servant à la performance. Elles sont moulées dans des matériaux mous, instables et organiques tels que la vaseline. L'action filmée est retransmise lors des expositions sur des écrans et les objets et sculptures sont laissés sur les lieux, 
transformant le lieu d'exposition en véritable sanctuaire dédié au culte du corps. Sur les deux écrans de Field Dressing (orifill), l'action retransmise montre l'artiste montant et descendant dans la pièce au-dessus d'une sculpture en vaseline, en forme d'emblème de terrain, dont il prélève la substance pour boucher ses orifices et faire de son corps un système clos. La sculpture participe littéralement à la construction de l'action et le corps est considéré comme un terrain qui peut être modifié et redessiné, un corps entièrement assujetti à la volonté humaine. Les objets sculptés utilisés dans cette action filmée font référence aux équipements servant à la construction du corps athlétique : les bancs de musculations, les haltères et les tapis de lutte. Les matériaux utilisés empruntent également beaucoup au vocabulaire sportif et plus précisément aux substances organiques ayant des répercussions sur le métabolisme comme les stéroïdes, le sucre ou les acides aminés. Dans ces objets, les équipements se superposent au métabolisme dans des objets construits à partir de substances biochimiques comme des haltères moulées de sucre ou de cire ou des machines mêlant le tapioca et le glucose. Blind Perineum, la plus longue des vidéos ( 87 minutes), montre Barney entrant dans la pièce réfrigérée de l'installation sculpturale TRANSEXUALIS, après avoir escaladé le plafond de la galerie. Radial Drill utilise les mêmes éléments mais dans des actions différentes, montrant que les deux vidéos n'ont pas pu se produire en même temps. En juxtaposant ces vidéos, Barney crée plusieurs zones temporelles, mais qui sont expérimentées en même temps par le spectateur. Cette désorientation est d'autant plus présente que les vidéos ne cessent de tourner en boucle. Le temps même devient rituel. Une tension est créée par la présence étrange des accessoires utilisés dans la vidéo, preuves d'un événement passé. L'assemblage du temps de la vidéo et du temps présent de l'expérience de l'installation instaure une frustration de la perception du spectateur de la notion de réel et d'imaginaire, de présent et de passé. Mais plus encore, Barney parvient à faire de l'espace dans lequel se déroule l'action un espace sculptural ${ }^{7}$.

7 Ces actions s'inscrivent dans la tradition de l'art de la performance et de la vidéo agrémentée de nouvelles iconographies, celles du sport et de la chirurgie, dont les actions qui en sont inspirées dégagent une atmosphère quasi-morbide dans la répétition de gestes traduisant un désir frustré et une virilité remise en cause. L'action de Barney est également influencée par l'esthétique télévisuelle qui anesthésie autant qu'elle spectacularise l'image du corps dans une certaine « société du spectacle ${ }^{8}$ ».

\section{Le corps comme véhicule de créativité et de sens}

8 Dans la création de Matthew Barney le corps devient une véritable analogie de la pratique artistique, à travers la performance, mais également à travers la perception de l'espace sculptural et muséal. Le déplacement des limites corporelles et humaines l'entraîne à redéfinir les limites de son art.

Le contrôle que Matthew Barney établit sur son œuvre est également imposé à son propre corps qui est pensé, comme le remarque Giovanna Zapperi, « comme un idéal de totalité, ce qui renvoie aux implications culturelles de son recours à la figure virile de l'athlète et, plus en général, à son discours sur le contrôle du corps ${ }^{9}$ ». En 2006, à l'exposition Drawing Restraint du San Francisco Museum of Modern Art, sont exposés trois dessins de graphite, vaseline et iode, fonctionnant à la manière de diagrammes conceptuels de la métaphore du système reproductif comme création artistique. Ils traduisent le système tripartite The Path, élaboré en 1990, et constitué des phases appelées "Situation, Condition, 
Production ». Ces notes et schémas préparatoires expriment le développement d'une énergie brute qui, une fois contrôlée et transformée, aboutit à une forme artistique. Ce concept émane du principe d'hypertrophie. L'un des exemples est celui du muscle qui se développe sous l'effort d'un poids. Appliqué à la création artistique, ce principe exprime l'idée que la force d'un travail repose dans la proportion de difficulté surmontée pour le créer $^{10}$. Le corps est envisagé comme un circuit, un organisme dont les mécanismes internes fonctionnent comme ceux d'une machine. Le corps est visualisé comme une machine et la machine comme un corps qui produisent toutes sortes de fluides abordant la dualité entre interne et externe.

10 Le processus de construction du corps est au cœur de la pratique de Matthew Barney. Cette démarche est peut-être la raison qui explique l'engouement et la fascination de la scène artistique new-yorkaise puis internationale pour ses travaux. Dès 1991, il séduit le milieu de l'art par le développement de sa mythologie personnelle qui lui permet d'élaborer un langage visuel figuratif après des décennies d'abstraction, mais qui maintient des aspects relativement abstraits dans sa signification. Il y associe des motifs récurrents tels que l'athlétisme, l'héroïsme, le transsexualisme et le contrôle du corps, et réintroduit l'image de l'artiste-héros ${ }^{11}$. La vidéo devient l'un des moyens d'expression les plus efficace et immédiat permettant au spectateur d'expérimenter l'œuvre en temps réel, selon les mêmes principes développés dans les théories phénoménologiques de Maurice Merleau-Ponty ${ }^{12}$. Le corps de l'artiste devient à la fois sujet et objet de l'œuvre. (Fig. 3) Dans l'épisode Radial Drill (1991), l'artiste gaine son corps dans une élégante robe de soirée, transformant son apparence dans un numéro de transgenre.

Fig. 3 : Matthew Barney, Radial Drill, 1988

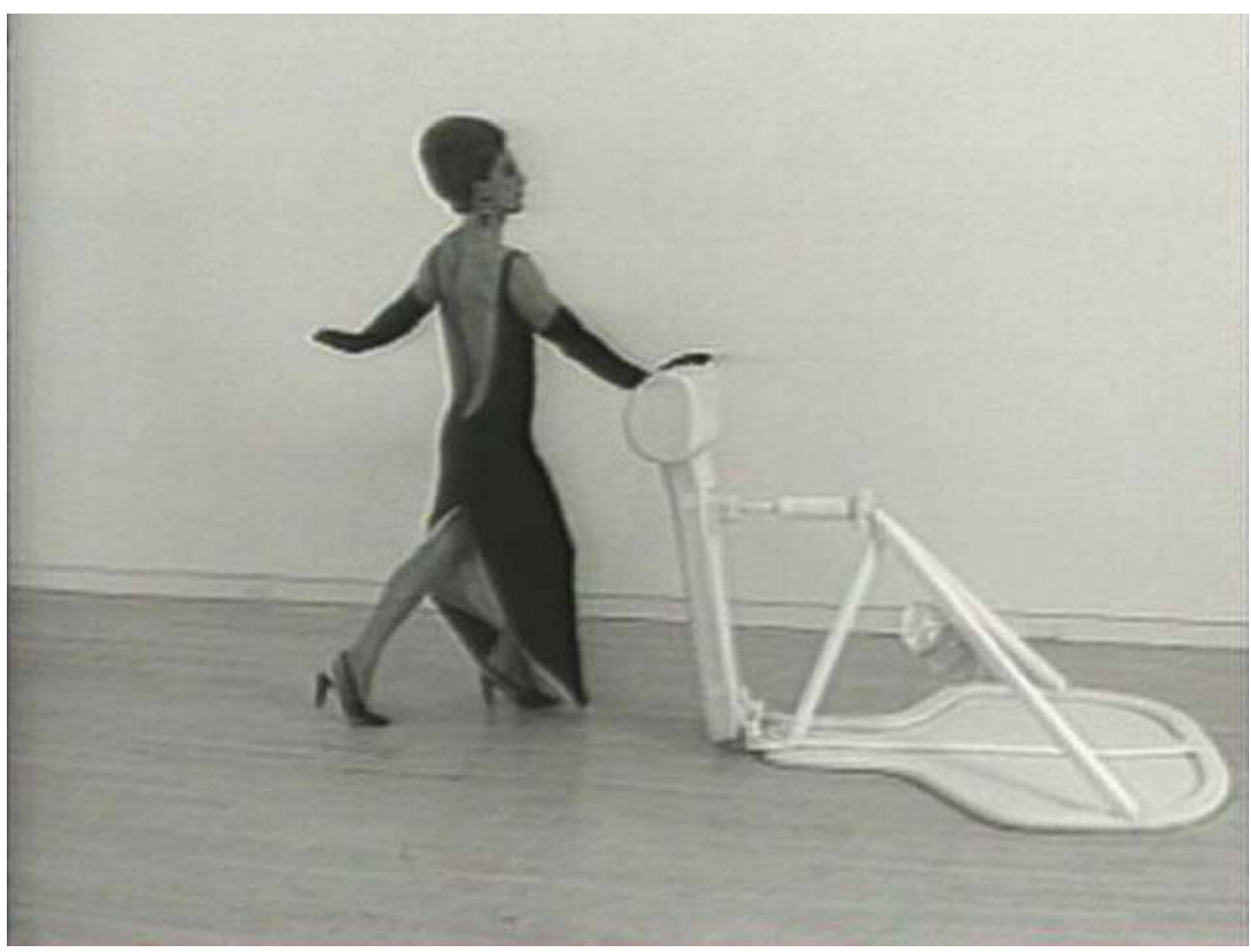

CAPTURE DE VIDÉo

Video: Peter Strietmann

Copyright Matthew Barney

Courtesy Gladstone Gallery, New York 
11 Pourtant, le spectateur ne participe pas à cette transformation, il n'en voit que la surface, à distance, sans participer à ce rituel ${ }^{13}$. Le corps de l'artiste est transformé par l'entraînement de préparation et la performance, mais est reçu plus comme une image que comme un processus qui montre le corps en tant que véhicule de l'effort et spectacle. Cette volonté d'ôter le corps et sa sexuation de tout contexte socioculturel, est avant tout un moyen de se focaliser sur la fonction formelle du corps comme véhicule de création. De la même façon que le personnage de l'athlète incarne un médium permettant d'expérimenter le développement d'une forme selon le principe d'hypertrophie superposé à la création artistique. Ainsi l'iconographie à laquelle Barney a recours et qui pourrait s'inscrire dans un discours social est envisagée d'un point de vue formel traduisant une dépolitisation de son art. Le principe de dépassement du corps continue d'être au centre de la pratique de Matthew Barney et se développe dans les cinq films du Cremaster cycle, dont chaque personnage principal doit surmonter des épreuves afin d'atteindre un but final.

\section{Questionnement identitaire et position d'auteur}

La remise en cause de la notion même d'auteur du statut d'artiste et par conséquent celui de créateur est certainement à l'origine de la volonté des artistes de réaffirmer leur identité.

Cette réaffirmation s'opère à différents niveaux : religieux, politiques ou encore sexuels, et s'exprime à travers l'usage de multiples médiums au sein de la génération d'artistes actuels dont fait partie Matthew Barney. L'identité et la réaffirmation du soi artistique se construisent par le biais d'un travail aux nombreuses références autobiographiques et d'un vocabulaire artistique singulier et parfois même à ce point imaginaire qu'il peut devenir quasiment étranger et incompréhensible du public et pourtant fascinant. Ce développement d'une mythologie personnelle ou individuelle est identifié dès la fin des années 1960, au cours d'expositions telles que celle d'Harald Szeemann, Quand les attitudes deviennent forme en 1969 puis la Documenta V de Cassel en 1972. Parallèlement, émerge la pratique désignée body work, évoquée par l'artiste et critique Wiloughby Shaps dans la revue Avalanche en $1970^{14}$. Ce renouveau du questionnement de l'identité d'artiste est également concomitant de l'avènement des gender studies développées dans les domaines de la sociologie et de la psychologie puis de l'histoire de l'art. Leurs études permettent une meilleure compréhension des interrogations sur le sexe et le genre qui réapparaissent chez les artistes des années 1990. Comme le souligne Giovanna Zapperi, après « le discours postmoderne de la fin de l'auteur [...] Barney réintroduit au contraire une position centrale de l'auteur ${ }^{15} »$. Au-delà de l'identité de l'artiste et de l'importance de son rôle, c'est « la recherche des principes fondamentaux de l'identité humaine ${ }^{16}$ ».

Mais la présence de l'artiste à travers l'image de la vidéo est remise en question par son absence même du lieu d'exposition. Il crée par son absence une relation de temps et d'espace à sa personne, car la vidéo retransmet une performance qui a eu lieu mais qui n'est plus, dans l'espace même où elle s'est déroulée. L'artiste impose sa présence à travers son absence ${ }^{17}$. Inspiré par les efforts physiques et psychologiques du joueur de football Jim Otto et du prestidigitateur Harry Houdini, Barney atteint «l'idéal de l'artiste " qui se travestit indéfiniment. Il exécute son œuvre en tant que genre et paradoxalement parvient à une quasi désindividualisation y perdant son identité propre. 
Dans ses actes, à travers lesquels Barney réaffirme la masculinité, il n'en donne qu'une lecture partielle puisqu'il remet en cause la masculinité comme genre qui possède une multitude d'interprétations et peut être vue comme une mascarade ${ }^{18}$. Dans le Cremaster Cycle, Barney devient tour à tour satyre, tueur en série, apprenti franc-maçon, Diva, cowboy ou magicien et fait appel au travestissement (costume, maquillage, prothèses) pour emprunter l'identité d'autrui, qu'il soit fictif, imaginaire ou historique et servir la narration.

Le titre même du cycle Cremaster fait référence à l'identité sexuelle, puisqu'au-delà de sa définition première (muscle qui régule le mouvement des testicules en fonction de stimuli externes) ce muscle, également présent dans l'organisme féminin, serait à l'origine de la détermination sexuelle chez le fotus. Il représente donc pour Matthew Barney un point de départ conceptuel, d'un potentiel d'énergie non déterminé, encore malléable, modifiable telle une matière à sculpter qui n'attend qu'à être mise en forme et avec laquelle tout est encore possible, en latence. Ce terme démontre la dualité des thématiques dans l'œuvre de Matthew Barney, à la fois érotique et biologique, féminin et masculin, sexe et genre. Dans le cycle Cremaster, parallèlement au questionnement de la différenciation autant que de celui de l'indifférenciation sexuelle, se posent la réaffirmation de l'identité artistique et la légitimation de la pratique de l'artiste par l'intervention d'autres artistes reconnus dans son travail.

16 Les personnages inventés par Barney, ces héros hybrides qui subissent d'incessantes modifications corporelles montrent que l'identité peut également s'exprimer par le corps, à la fois machine de notre monde post-humain et champ de bataille de toutes les expressions humaines ${ }^{19}$. Bien que l'identité sexuelle soit centrale dans la problématique de Barney, la multiplicité des personnages imaginaires et travestis témoigne des possibilités infinies de l'individu à se réinventer dans une quasi-schizophrénie.

17 Cette manipulation dans sa totalité soulève un problème récurrent de l'œuvre de Matthew Barney, celui de la volonté de contrôle à la fois sur le corps et dans sa conception comme un système dans sa totalité, sur l'image de représentation dans sa perfection et sur l'identité empruntée.

\section{Entre culture post-humaine et biomorphisme}

Les personnages présents dans l'œuvre de Matthew Barney sont presque tous ce que l'on pourrait appeler des mutants, des êtres dont le devenir est post-humain et qui s'inscrivent dans la culture émergente du « techno-body ${ }^{20}$ ». 
Fig. 4 : Matthew Barney, CREMASTER 4, 1994

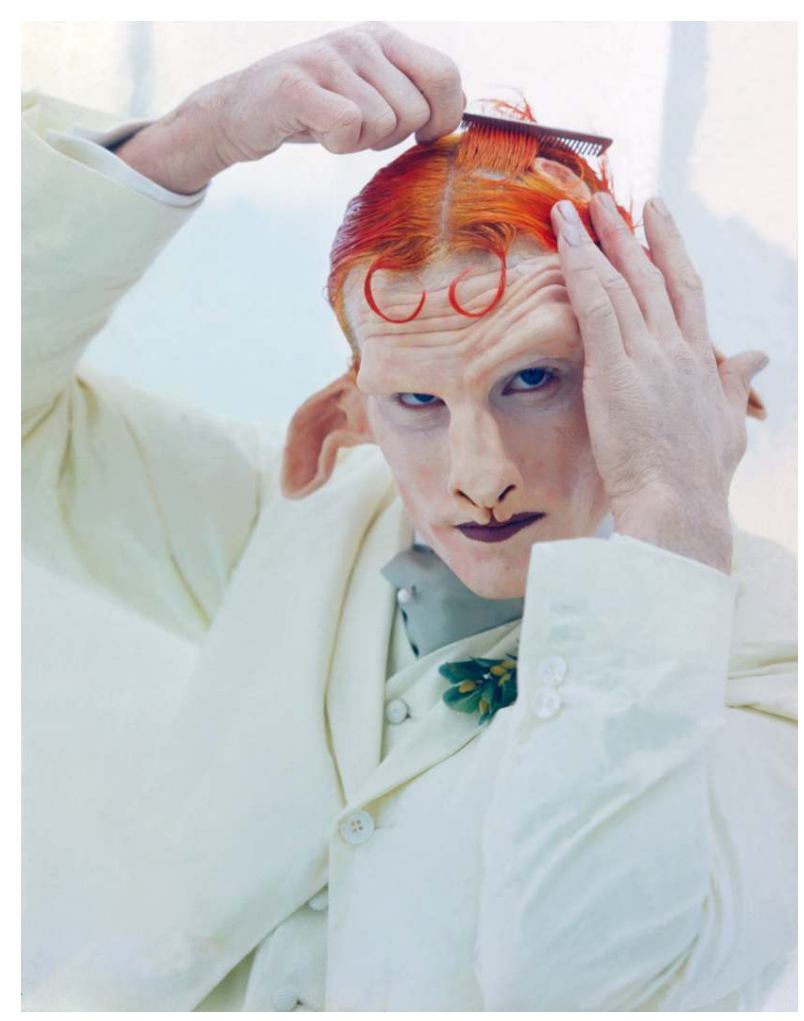

\section{PHOTOgRAPHIE DE fiLM}

(C)1994 Matthew Barney

Photo: Michael James O'Brien

Courtesy Gladstone Gallery, New York

(Fig.4) Dans Cremaster 4, le personnage hybride du Loughton Candidate doit son apparence en grande partie aux prothèses et au maquillage, mais également à son travestissement en satyre-dandy, selon le principe d'après lequel le corps et l'esprit veulent surpasser leur condition. Le Loughton Candidate tente de se maintenir dans un état d'indétermination sexuelle, entraînant la construction d'un corps dans lequel se côtoient des éléments à la fois féminins et masculins, mais aussi humains et animaux. Cette volonté apparaît dès le début du film lorsque, face au miroir, il découvre ses embryons de cornes, qu'il doit maintenir dans cet état s'il veut conserver son statut intermédiaire. Tout comme celle des trois Faeries qui l'accompagnent, son apparence, bien que modifiée à l'aide de maquillage, prothèses et costumes, pourrait relever d'une intervention chirurgicale ou d'une modification génétique s'inscrivant dans l'esthétique d'un imaginaire mythique et monstrueux. Barney a d'ailleurs parfois été comparé à un néo-surréaliste étrange, jouant avec les peurs humaines des modifications génétiques et des monstres mutants ${ }^{21}$. Il développe un fantasme utopique d'un monde développé à partir d'un système sexuel pouvant être modifié à merci. Devant cette profusion d'espèces étranges et souvent inconnues qui défilent sur les écrans et apparaissent sur les photographies, l'art ne peut que rencontrer la biologie et l'artiste devient le biologiste créateur de ces êtres visionnaires qui viennent contrarier la signification du terme nature pour mieux nommer la folie de la génération cyber-biologique ${ }^{22}$. Ces corps qui n'étaient que pure anticipation et imaginaire dans les décennies 1960 et 1970, font désormais partie de notre environnement contemporain et de notre monde post-humain ${ }^{23}$, des corps modifiés par 
les nouvelles technologies, siliconés, améliorés à l'aide de prothèses qui relèvent à la fois du réel et de l'artificiel comme du biologique et du mécanique ${ }^{24}$.

\section{Du genre aux genres}

Bien qu'étant attirantes visuellement parlant, les créatures et les transformations corporelles de Matthew Barney traitent de thèmes qui relèvent de dualités telles que le genre et le sexe, le masculin et le féminin.

Tout comme les autres paradoxes de son œuvre, la relation de la masculinité au corps et à la sexualité est un sujet que Matthew Barney semble aborder ou parfois refuser. Dans les récentes recherches effectuées sur les gender studies, est abordée la possibilité que l'identité du genre et la sexualité puissent être vécues et pensées séparément. C'est dans ce contexte que les œuvres de Matthew Barney peuvent être analysées et interprétées. Mais au-delà de la thématique du genre, il faut peut-être également envisager dans une certaine perspective le manque de genre, plus simplement. Les créatures de Matthew Barney ont souvent été décrites comme étant hypersexuées ${ }^{25}$, pourtant la plupart n'ont pas de parties génitales apparentes; ce sont des corps sans organes, des machines conduites par le désir et à leur tour productrices de désir, comme l'envisageaient Deleuze et Guattari ${ }^{26}$. C'est justement cette absence d'organes dans l'œuvre de Matthew Barney qui empêche toute symbolique phallique et tourne en ridicule la représentation de la masculinité : les trois fées du Cremaster 4 apparaissent tantôt nues et asexuées, tantôt vêtues de robes bouffantes jaunes. Le Loughton Candidate et le Géant du Cremaster 5 (fig. 5) ne laissent apparaître qu'un scrotum atrophié. L'artiste utilise son propre corps pour représenter des personnages masculins émasculés. Cette représentation de la masculinité renverse quelque peu ou, en tout cas, contrebalance la volonté de pouvoir et de contrôle qui existe chez l'artiste. Finalement, les deux seules scènes dans toute l'œuvre de l'artiste où est distinguable le motif du pénis sont celle de la reproduction dans Cremaster 2, mais qui appartient à un corps déshumanisé, sans visage, automatisé, qui se désintègre littéralement. 
Fig. 5 : Matthew Barney, CREMASTER 5, 1997

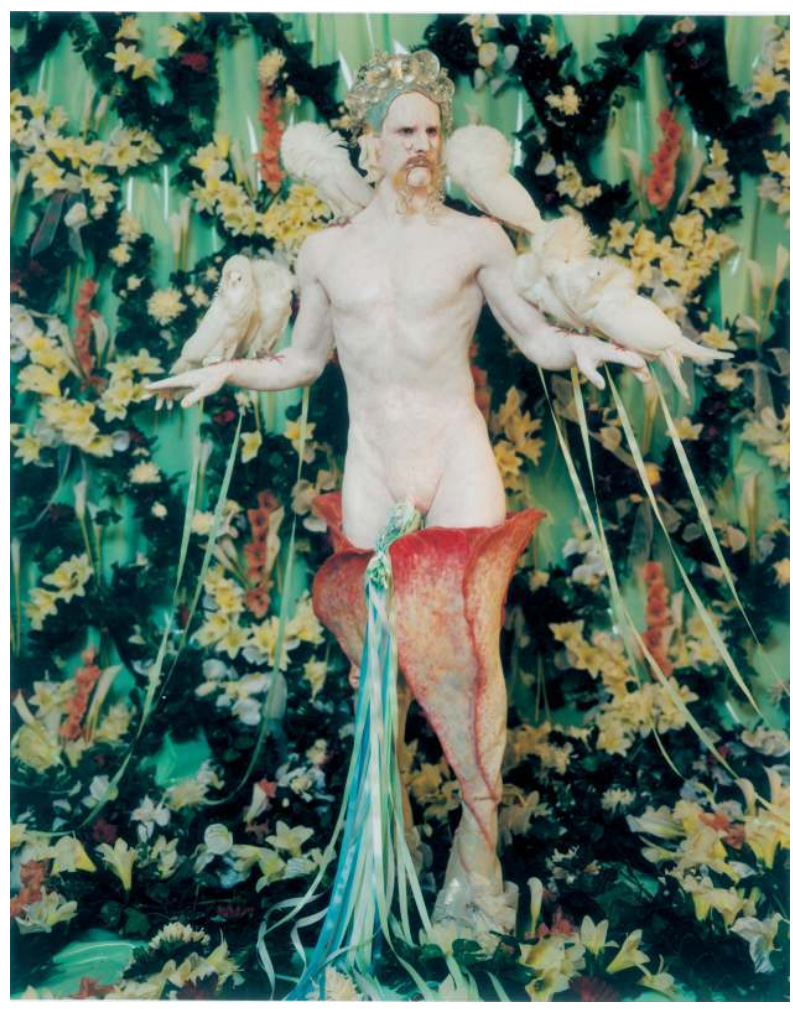

\section{PHOTOgRAPHIE DE fiLM}

(C)1997 Matthew Barney

Photo: Michael James O'Brien

Courtesy Gladstone Gallery, New York

Matthew Barney refuserait donc finalement toute implication dans le masculin comme dans le féminin, pour préférer rester dans cette zone indéterminée qu'il appelle zone de pleine potentialité, une zone qui d'un point de vue corporel serait androgyne. C'est également cette identité troublée qui génère la fascination autant que l'anxiété. Il refuse d'adopter une relation mécanique entre la sexualité et le genre, mais conçoit même une identité indifférenciée du genre et de la sexualité et ainsi un espace de toutes les possibilités fictionnelles.

\section{BIBLIOGRAPHIE}

\section{Ouvrages :}

DEBORD, Guy, La Société du Spectacle, Paris, Buchet-Chastel, 1967.

DELEUZE, Gilles, et GUATTARI, Félix, Anti-Oedipe : Capitalisme et schizophrénie, Paris, Minuit, 1994.

GIONI, Massimilio, Matthew Barney, Milan, Electa, 2007 
MAHON, Alyce, Eroticism and Art, New York, Oxford University Press, 2005, p. 279-281.

MERLEAU-PONTY, Maurice, Phénoménologie de la perception, Paris, Gallimard, (1945), 2001

PARFAIT, Françoise, Vidéo : un art contemporain, Paris, Regard, 2001, p. 245.

PERCHUK, Andrew, The Masculine Masquerade, Cambridge, MIT Press, 1995.

Articles :

BROD, Harry, "Masculinity as Masquerade", in The Masculine Masquerade, Cambridge, MIT Press, 1995

DAGEN, Philippe, « L'art total de Matthew Barney », in Le Monde, 13 octobre 2002, p. 21

GOODEVE Thyrza-Nichols, « Matthew Barney 95. Suspension (cremaster), Secretion (pearl), Secret (biology)», in Parkett nº 45, Zurich, 1995, p. 67-69.

JONES, Amelia, « Presence in abstentia : experiencing performance as documentation », in Art Journal, vol. 56, n 4, Hiver 1997, p. 11-18.

KOTZ, Liz, "Video : process and duration", in Acting Out (The Body in Video : Then and Now), Londres, Royal College of Art, 1994, p. 17-26.

MORGAN, Stuart, « Of Goats and Men », in Frieze n 6, Janvier-Février 1995, Londres, p. 34-38.

SEWARD, Keith, « Matthew Barney and Beyond », in Parkett n 45, Zurich, 1995, p. 58-61.

SPECTOR, Nancy, « Performing the body in the 1970's ", in Rose is a rose is a rose: Gender performance in Photography, New York, Guggenheim Museum Publications, 1997.

TROTMAN, Nat, "Ritual space/ Sculptural Time", in All in the present must be transformed: Matthew Barney and Joseph Beuys, New York, Guggenheim Museum Publications, 2007, p. 145.

ZAPPERI, Giovanna, « Matthew Barney : Systèmes de production », in Pratiques : Réflexions sur l'art $n^{\circ}$ 17, Hiver 2006, Rennes, Presses Universitaires de Rennes, p. 53-68

\section{Catalogue d'exposition :}

Matthew Barney: New Work, San Francisco Museum of Modern Art, San Francisco, 1991

Périls et colères, (CAPC-Musée d'art contemporain, Bordeaux, 22 mai- 6 sept. 1992), Bordeaux, CAPC-Musée d'art contemporain, 1992.

Post-human, Musée d'art Moderne, Lausanne, FAE Musée d'art contemporain, 1993.

\section{NOTES}

1. Giovanna Zapperi, « après le discours postmoderne de la fin de l'auteur [...] Barney réintroduit au contraire une position centrale de l'auteur", in Giovanna Zapperi, "Matthew Barney: Systèmes de production ", in Pratiques: Réflexions sur l'art n ${ }^{\circ}$ 17, Hiver 2006, Rennes, Presses Universitaires de Rennes, p. 53-68.

2. Dan Cameron, Périls et Colères, Bordeaux, Musée d'art contemporain, 1992.

3. Cf. Matthew Barney: New Work, San Francisco Museum of Modern Art, San Francisco, 1991.

4. Philippe Dagen, «L'art total de Matthew Barney », in Le Monde, 13 octobre 2002, p. 21.

5. Stuart Morgan, «Of Goats and Men », in Frieze n6, Londres, Janvier-Février 1995, p. 34-38.

6. Andrew Perchuk, The Masculine Masquerade, Cambridge, MIT Press, 1995.

7. Nat Trotman, "Ritual space/ Sculptural Time", in All in the present must be transformed: Matthew Barney and Joseph Beuys, New York, Guggenheim Museum Publications, 2007, p. 145. 
8. Guy Debord, La Société du Spectacle, Paris, Buchet-Chastel, 1967.

9. Op. Cit Giovanna Zapperi, p. 59.

10. Keith Seward, «Matthew Barney and Beyond», in Parkett $n^{\circ} 45$, Zurich, 1995, p. 58-61.

11. Op. Cit Giovanna Zapperi, p. 53-68.

12. Maurice Merleau-Ponty, Phénoménologie de la perception, Paris, Gallimard, (1945), 2001.

13. Liz Kotz, "Video: process and duration", in Acting Out (The Body in Video: Then and Now), Londres, Royal College of Art, 1994, p.17-26.

14. Nancy Spector, "Performing the body in the 1970's", in Rose is a rose is a rose: Gender performance in Photography, New York, Guggenheim Museum Publications, 1997.

15. Op. Cit Giovanna Zapperi, p. 53-68.

16. Op. Cit Dan Cameron.

17. Amelia Jones, «Presence in abstentia : experiencing performance as documentation », in Art Journal, vol. 56, n 4, Hiver 1997, p. 11-18.

18. Harry Brod, "Masculinity as Masquerade", in The Masculine Masquerade, Cambridge, MIT Press, 1995.

19. Massimilio Gioni, Matthew Barney, Milan, Electa, 2007.

20. Alyce Mahon, Eroticism and Art, New York, Oxford University Press, 2005, p. 279-281.

21. Ibid. p. 279-281.

22. Thyrza-Nichols Goodeve, «Matthew Barney 95. Suspension (cremaster), Secretion (pearl), Secret (biology)», in Parkett nº 45, Zurich, 1995, p. 67-69.

23. «Les artistes ne pourront plus s'engager simplement dans la redéfinition de l'art. Dans l'ère posthulaine, ils seront sans doute impliqués également dans une nouvelle redéfinition de la vie ", in Jeffrey Deitch, Post-human, Musée d'art Moderne, Lausanne, FAE Musée d'art contemporain, 1993.

24. Massimilio Gioni, Matthew Barney, Milan, Electa, 2007.

25. Françoise Parfait, Vidéo : un art contemporain, Paris, Regard, 2001, p. 245.

26. Gilles Deleuze et Félix Guattari, Anti-〔Edipe : Capitalisme et schizophrénie, Paris, Minuit, 1994.

\section{RÉSUMÉS}

Dès ses premières œuvres, l'artiste américain Matthew Barney exhibe son corps d'athlète dans des épreuves physiques d'endurance et de douleur témoignant d'un contrôle et d'une puissance sur le corps de l'artiste. Au-delà de l'identité de l'artiste, c'est «la recherche des principes fondamentaux de l'identité humaine» (Dan Cameron, Périls et Colères, Bordeaux, Musée d'art contemporain, 1992). Dans chacune de ses œuvres Barney se travestit et adopte l'identité d'un personnage afin d'exécuter sa performance dans ses deux séries Drawing Restraint (1987-2009) et The Cremaster Cycle (1992-2002). Il exécute son œuvre en tant que genre dans une quête de « l'idéal de l'artiste» qui se travestit indéfiniment. Barney ne donne qu'une lecture partielle de sa masculinité puisqu'il remet en cause le genre par une multitude d'interprétations telle une mascarade(Harry Brod, « Masculinity as Masquerade», in The Masculine Masquerade, Cambridge, MIT Press, 1995). Cette réinterprétation du soi artistique témoigne des possibilités infinies de l'individu à se réinventer dans une quasi-schizophrénie. 


\section{AUTEUR}

\section{MARIE-LAURE DELAPORTE}

Doctorante, chargée d'enseignement en histoire de l'art contemporain à l'Université de ParisOuest-Nanterre-La-Défense. Elle mène des recherches sur l'œuvre de Matthew Barney et la notion d'œuvre d'art total. Elle a été commissaire de l'exposition ÉpHémérité/ÉffemMinité, à l'espace « 6 bis », Paris, en avril et mai 2009. Sujet de thèse : L'artiste à la caméra : pour une hybridation des genres artistiques (des années 1960 à nos jours).

Publications :

«Intérieur/Extérieur : les lieux de Lucian Freud », in Hors-série Lucian Freud, éditions Beaux-arts Magazine, mars 2010, p. 24-31.

"L'artiste et ses modèles », in Hors-série Lucian Freud, éditions Beaux-arts Magazine, mars 2010, p. 32-37.

"Giacometti : portrait de famille ", in Hors-série Giacometti, à la Fondation Beyeler, éditions Beauxarts Magazine, mai 2009, p. 8-11.

Texte d'introduction du catalogue de l'exposition 14-21: art contemporain iranien, à l'espace Kiron, Paris, février 2009.

«La jeune scène chypriote ", in Beaux-arts Magazine $\mathrm{n}^{\circ}$ 293, novembre 2008, p. 160-167. 